\title{
HISTORISCHE UND GEISTIGE GRUNDLAGEN DES DEMOKRATISCHEN GEDANKENS IN SPANISCHAMERIKA
}

\author{
Von FrIEDRICH WEHNER
}

\section{Demokratie als notwendiges und generelles Problem}

Die lateinamerikanischen Länder überraschen immer wieder mit Vorgängen auf der politischen Bühne, die nur schwer durchschaubar sind; Motive und Hintergründe sind in breiteren Kreisen unbekannt. Hierzu gehören vor allem das fortgesetzte Auftreten von diktatorischen Militärregierungen und das offenbare Nichtfunktionieren der Demokratie. Es ist zwar oft von Demokratie die Rede, in der Praxis ist sie nicht vorherrschend ${ }^{1}$. Wenn behauptet wird, daß die Demokratie „está presente en el cotidiano quehacer, responsabilizando al ciudadano y garantizándole su participación en la elaboración de los destinos nacionales", so klingt das wunderschön, mit der Wirklichkeit stimmt es nicht überein. $D a$ ist es schon wahrhaftiger, wenn der frühere Präsident von Venezuela, Rómulo Bétancourt, feststellt: La inestabilidad política y la falta de solidez de las instituciones democráticas es otra constante en la región ${ }^{2}$.

Es muß auffallen, daß die Thematik der Demokratie oft behandelt wird, ohne die geschichtliche Entwicklung und die geistige Herkunft genügend zu würdigen und $\mathrm{zu}$ fragen, ob und inwieweit der Charakter der heutigen Staaten und ihrer Bewohner auch aus den Umständen zu erklären ist, unter denen vor rund 150 Jahren die Bildung der selbständigen Staaten als Nachfolger der iberischen Kolonialherrschaft erfolgte.

In diesem Sinne soll hier der Versuch unternommen werden, die komplexe Problematik der Demokratie in den spanischamerikanischen Ländern Lateinamerikas aus hiesiger Sicht analysierend zu skizzieren. Da diese Staaten in ihren Verfassungsmodellen sich stark an westeuropäische Ideen und das USA-Vorbild anlehnen, ist eine Orientierung an diesen Vorbildern und dem ihnen zugrundeliegenden Geistesgut erforderlich.

Angesichts der weltweiten Durchsetzung des demokratischen Gedankens muß man auch für Spanischamerika davon ausgehen, daß nur eine irgendwie geartete demokratische Entwicklung und Gestaltung des politischen, wirtschaftlichen und sozialen Lebens zeitgemäß sein kann. „Einen anderen Statstyp (als die Demokratie)... gibt es heute nicht mehr ${ }^{3}$." Dies muß mindestens im Sinne einer aktiven Anteilnahme aller Bevölkerungsteile am öffentlichen Geschehen gelten. Dieser Satz mag angesichts des häufigen Vorkommens von Militärregierungen befremdlich klingen - er gilt grundsätzlich aber auch für ganz Lateinamerika.

Die Demokratie hat sich für unser säkulares Zeitalter als diejenige „Herrschaftsund Lebensform" "4 erwiesen, aus der allein eine Legitimierung weltlicher Regierungsgewalt abgeleitet werden kann. Nach Erlangung der staatlichen Unabhängig-

\footnotetext{
1 So u. a. Carlos Pisano Fischer, El Poder Ejecutivo en las Constituciones Latinoamericanas. Santiago de Chile 1968, S. 133. - Pablo Gonzalez Casanova, La democracia en México. - México D. F. 1965 , S. 6, 11.

2 In einer 1965 gehaltenen Rede unter dem Titel: Revolución Democrática en América Latina.

3 Herbert Krüger, Allgemeine Staatslehre. - Stuttgart 1964. - S. 368.

4 Carl Joachim Friedrich, Demokratie als Herrschafts- und Lebensform. - 2. Aufl., Heidelberg 1966.
} 
keit in den Jahren 1810 bis 1825 war es naheliegend und historisch auch richtig, zum demokratischen Konstitutionalismus überzugehen. Das spanischamerikanische Problem liegt darin, daß zwar demokratische Verfassungen formuliert wurden, daß aber nicht ernsthaft genug geprüft wurde, welche der mancherlei Spielarten sich eignen und ob anderswo unter anderen Verhältnissen und aus anderem Geiste gebildete Formen auch auf die spanisch-amerikanischen Umstände passen würden. Bisher haben die übernommenen Formen den politischen Prozeß nur in den wenigsten Fällen in Gang zu bringen vermocht, es kommt meistens nicht zu einem rechten „juego democrático“. Es ist daher zu untersuchen, welche Bedeutung die übernommenen Ideen westeuropäischer und USA-Herkunft für die Unabhängigkeitskämpfe gehabt haben und warum sie nur eine zweifelhafte Wirkung ausüben konnten. Der Ausgangspunkt der Überlegungen darf dabei nicht sein, daß die übernommenen Modelle in jedem Falle "richtig“ und die politische Wirklichkeit „falsch“ sei. Vielmehr ist aus der Wirklichkeit abzulesen, inwiefern die vorhandenen demokratischen Verfassungen nicht mit der spanischamerikanischen Existenz und ihrem Selbstbewußtsein, ihrem historischen Werden und ihrer Ideologie übereinstimmen.

Eine Untersuchung über "die" Demokratie in Spanischamerika gerät in die Gefahr, allzusehr zu generalisieren. Von Spanischamerika aus betrachtet, erscheinen die nationalen Unterschiede größer und gewichtiger als für den Außenstehenden. Alle Verfassungen sind in ähnlichem Tenor und Wortlaut formuliert worden - alle enthalten gleiche Merkmale demokratischer Systeme, wie Garantie der individuellen Grundrechte, freie Wahlen oder das Prinzip der Gewaltentrennung. Der chilenische Rechtsgelehrte Carlos Pisano Fischer stellt fest: „Hay en Sudamérica ... una unidad esencial que nos permite hablar de un derecho constitucional hispano-americano“, - und: „La Diversidad de sus paises no rompe la unidad hispano-americana"5. F. García Calderón geht in einem kurz vor dem Ersten Weltkrieg geschriebenen Buche über die „Lateinamerikanischen Demokratien“ von dem "einförmigen Verlauf der Ereignisse" aus und kommt von da aus zur Behandlung der einzelnen Länder 6 . - wie wohl grundsätzlich die nationalen Differenzierungen erst auf der Basis der Gemeinsamkeiten voll verständlich werden. Die kulturelle und sprachliche Verwurzelung in der spanischen Geisteswelt und Tradition, die gleiche Herkunft aus dem spanischen Kolonialreich, die häufig angesprochene „Unidad del Pensamiento“, die Charakterzüge, die Ähnlichkeiten im gesellschaftlichen System und in den noch stark semikolonialen Wirtschaftsformen, die Fortdauer eines gewissen intellektuellen Kolonialismus, diese insgesamt gesehen „estructura social totalmente heterogénea“ gelten für alle Länder, wenn auch mit Gradunterschieden, und sie werden auch in vielen Studien als gegeben vorausgesetzt. In diesem Sinne wird auch der Ausdruck „el estado latino-americano“ generalisierend verwandt ${ }^{7}$.

5 C. Pisano Fischer, vgl. Anm. 1). - S. 125.

6 F. García Calderón, Die Lateinischen Demokratien Amerikas. - Deutsch bei K. F. Koehler, Leipzig 1913, S. 2.

arcos Kaplan in einem Vortrag über "La Estructura del Estado en el Desarrollo de América Latina". - In: Perspectivas del Progreso, hrsg. v. d. Friedrich-Ebert-Stiftung, Hannover 1969, S. $105 \mathrm{ff}$. 


\section{Von der Monarchie zur Oligarchie}

\section{Ausklang der Monarchie}

Nach einer dreihundertjährigen Tradition monarchischer Regierungen der spanischamerikanischen Provinzen ist es auffällig, wie rasch sich dies bisher einheitlich regierte Gebiet in selbständige Staaten auflöste ${ }^{8}$ und wie schnell der monarchische Gedanke fallengelassen wurde, um republikanisch freiheitlichen Vorstellungen Platz zu machen.

In den ersten Jahren der Unabhängigkeitsbewegungen von 1810 bis 1825 wurde nicht generell an eine Preisgabe der monarchischen Idee gedacht. Ein Beispiel früher republikanischer Vorstellungen ist der Verfassungsentwurf des'Mexikaners José María Morelos y Pavón in der Erklärung von Apatzingán vom 22. Oktober 1814 , in der als oberstes Organ ein Dreierrat vorgesehen war. Einen stärkeren Einfluß hatte die spanische Verfassung von Cádiz vom 19. März 1812, an deren beschlußfassender Versammlung auch einige Vertreter spanisch-amerikanischer Gebiete (damals noch Vizekönigreiche) teilgenommen hatten. Diese Verfassung fand weitgehende Zustimmung; ihr bestimmendes Motiv war die Treue zu dem angestammten Herrscherhause gegenüber dem Usurpator Napoleon. Die Verfassung enthält die Bestimmungen einer konstitutionellen Monarchie für das gesamte Gebiet Spaniens und Spanischamerikas und bescherte den Bewohnern Amerikas die langersehnte Erfüllung zweier wichtiger Wünsche: die Gleichstellung von Spaniern und Spanischamerikanern (Kreolen) sowie die parlamentarische Mitwirkung an der Regierung. Sie stellte somit den ersten Versuch dar, die spanische Tradition mit liberalen Ideen zu verknüpfen - sie wollte Reformen im Rahmen der traditionellen Monarchie verwirklichen.

Die radikale Wende in der Einstellung zur Monarchie entsprang nicht zuletzt der brüsken Art, mit der König Ferdinand VII. nach Rückkehr aus der Verbannung nach Besiegung Napoleons die Verfassung von Cádiz bereits mit Dekret vom 4. Mai 1814 für null und nichtig erklärte. Dieses Verbot mit Androhung der Todesstrafe mußte wie ein Schock auf die Gutwilligkeit der Spanischamerikaner wirken und die Gefühle für die Monarchie erkalten lassen. Die brutale Form der Aufhebung der Verfassung und die damit ausgelöste Enttäuschung trugen wesentlich dazu bei, daß sich die Gemüter den republikanisch-demokratischen Ideen und der Bildung unabhängiger Nationalstaaten zuwandten. Simón Bolívar hielt bereits 1815 eine "monarquía universal de América" für ebenso unmöglich wie eine einzige Republik ${ }^{9}$.

Es erwies sich auch, daß die monarchische Idee in Spanischamerika nicht so tief verwurzelt war wie in den Herzen vieler Spanier selbst. Die rasche Abwendung der Spanischamerikaner von dem monarchischen Gedanken ist ein Indiz dafür, $\mathrm{d} a ß$ entscheidende innere Voraussetzungen gefehlt haben müssen. Im Verlaufe der dreihundertjährigen Regierung der spanischen Krone hatte sich offenbar eine unerschütterliche monarchische Tradition nicht bilden können. Die Spanischamerikaner erlebten die Monarchie nur in Gestalt der von der Krone entsandten Statthalter und Beamten. Die direkten Kontakte zum Herrscherhause waren ver-

$8 \mathrm{Vgl}$. die Darstellung von Inge Wolff, Desintegration und Staatenbildung in Hispanoamerika 1810-1825. - In: F. Wehner (Hrsg.), Idee und Wirklichkeit in Iberoamerika, Beiträge zur Politik und Geistesgeschichte. Hamburg 1969, S. $41 \mathrm{ff}$.

9 In der Carta de Jamaica, Ausgabe Caracas 1965, S. 32. 
hältnismäßig lose und schwach. Der König selbst war zu weit entfernt, schon die damaligen Verkehrsverhältnisse verhinderten das persönliche Auftreten des Königs in seinen Provinzen. In den letzten Jahrzehnten des 18. Jahrhunderts hatten sich zudem Gefühle der Abneigung gegen das königliche Regime wegen ständiger Zurücksetzungen hinter den aus Spanien entsandten Beamten erhärtet $^{10}$. Günter Kahle weist darauf hin ${ }^{11}$, daß in México nach den bourbonischen Reformen von 1769 Rekrutierte nur widerwillig dienten. Sie konnten nur schwer begreifen, daß es eine Ehre sein sollte, dem König von Spanien im Waffenhandwerk zu dienen nur deshalb, weil vorgesetzte Behörden es so behaupteten. Ebenso wie solche Rekrutierungen als ungerecht und unverständlich erscheinen mußten, wirkten sich zusätzlich die in den letzten Jahrzehnten an Armee und Miliz reichlich vergebenen Sonderrechte (fueros) zersetzend auf die normale Gerichtsbarkeit aus und erschütterten ebenfalls das Ansehen der königlichen Verwaltung, die an Respekt und Prestige verlor ${ }^{12}$. Letzten Endes war es aus der starken klerikalen Tradition heraus aber auch wohl das Gefühl, daß ein Monarch einem angestammten Herrscherhause „von Gottes Gnaden“ entstammen müsse. Mindestens hätte sich vorher ein herausragender Adel gebildet haben müssen ${ }^{13}$. Aus welcher sozialen Schicht hätten neue Dynastien entstehen können? Auch Simón Bolívar hat sich nicht entschließen können, die ihm angebotene Krone anzunehmen.

Als sich die Vertreter eines Teils der jungen Staaten zu der von Bolívar einberufenen Konferenz von Panamá im Jahre 1826 zusammenfanden, gab es nur noch Republiken. Einzelne Versuche und mancherlei Überlegungen, dennoch Monarchien zu errichten, hatten nur kurzfristige Erfolge. In México hatte sich Augustin de Iturbide am 18. Mai 1822 zum Kaiser ausrufen lassen, nachdem Bestrebungen konservativer Kreise gescheitert waren, dem spanischen König Ferdinand VII. die Kaiserkrone anzutragen. Bereits im März 1823 mußte Iturbide wieder entsagen. Ebenso scheiterte der Versuch europäischer Mächte, 1865 dem Lande México einen europäischen Fürsten als Kaiser aufzuzwingen; diese Episode endete mit der Erschießung Maximilians im Jahre 1867.

Im außerspanischen Amerika gab es in Haiti einige Ansätze zu monarchischen Perioden, die aber ohne nachhaltige Wirkung vorübergingen. - Anders verliefen die Ereignisse zunächst in Brasilien. Durch Ubersiedlung des königlich portugiesischen Hofes nach Rio de Janeiro im Jahre 1807 fand eine organische Fortsetzung der Monarchie auf amerikanischem Boden statt, seit 1822 in Form eines konstitutionellen Kaiserreiches, das 1889 gestürzt wurde.

\section{Anarchie und Diktatur}

Die republikanische Staatsform steht zunächst nur im formalen Gegensatz zur Monarchie und bedeutet die Fundierung der Macht im Volke und für das Volk, und damit die Ablehnung einer nicht abwählbaren sich auf Gott berufenden Dynastie. Ihre staatsrechtliche Ausgestaltung sollten die jungen Republiken durch Übernahme demokratischer Verfassungen erhalten.

\footnotetext{
10 Instruktive Beispiele von Außerungen auszugsweise in: Richard Konetzke, Lateinamerika seit 1492. S. $36 \mathrm{ff}$. (Quellen und Arbeitshefte, Nr. 4258. - Klett Verlag Stuttgart).

11 Günter Kahle, Militär und Staatsbildung in den Anfängen der Unabhängigkeit Mexikos. - Köln/Wien 1969. - S. 35

12 id., S. 44.

13 Vgl. die đußerung von Bolívar zu San Martín vom 26. Juli 1822. In Konetzke, vgl. Anm. 10), - S. 61.
} 
In der politischen Praxis wird es immer eine Diskrepanz geben zwischen geschriebenem Recht und politischer Wirklichkeit, die stets in einem fortschreitenden Entwicklungsprozeß begriffen ist. Verfassungstext und politische Wirklichkeit stehen in einem ständigen Spannungsverhältnis, wobei die Verfassung die Leitmotive gibt, die in einem bestimmten Zeitpunkt aufgestellt worden sind. Diesen "Sachverhalt der Diskrepanz"14 gibt es überall, ohne daß er allerdings ein so schwerwiegendes Problem sein muß wie in Spanischamerika ${ }^{15}$. Es fehlte von vornherein an der inneren Übereinstimmung zwischen den Zielsetzungen der Verfassungen als „instruments of government" und dem Willen der Machthaber wie auch des Volkes, soweit von einem „Volke“ zunächst überhaupt die Rede sein konnte. Die Verfassungen der spanischamerikanischen Republiken in ihrer Eigenschaft als institutionelle Nachfolger der monarchischen Form blieben hoffnungslos ohne Einfluß - sie sind es zu einem hohen Grade auch heute noch. Sie haben bisher überwiegend in ihrer Funktion versagt, Ordnung und Recht zu garantieren.

Anstelle einer funktionierenden Demokratie mit Garantierung der individuellen Rechte und einem ausgewogenen Verhältnis zwischen Exekutive, Legislative und Justiz etablierte sich sogleich ein schrankenloses Überwiegen der Exekutive in Form einer systemlosen diktatorischen Gewaltherrschaft als Caudillotum, einer spezifisch spanischamerikanischen Form personalistischer Führung. Die Verfassung als Institution geriet dabei in den Schatten ehrgeiziger macht- und einflußhungriger, häufig auch materialistisch und egoistisch handelnder Diktatoren ${ }^{16}$.

Ebenso wie sich die Institution der Verfassung als zu schwach erwies gegenüber der Entfesselung personalistischer und individualistischer Willkür, versagte auch das überlieferte Gefüge der spanischen Kolonialzeit. Es vermochte nicht, für den Übergang zu neuen Formen noch einen gewissen Halt zu bieten. Die vorhandenen Verwaltungseinrichtungen und traditionellen Werte wurden rasch verwirtschaftet und gute Kräfte im Zuge der Unabhängigkeitskämpfe und der Abschüttelung der kolonialen Bevormundung verjagt. Anarchische und chaotische Zustände waren die Folge. Auch im Heere verfielen die konservierenden Merkmale der Tradition auch das Herr erfüllte seine Funktion als fester Übergangsfaktor nicht, im Gegenteil gewannen auch dort die egoistischen und zerstörerischen Ambitionen die Oberhand. Unwürdige Methoden der Rekrutierung und der Behandlung der indianischen Handwerker und Landarbeiter mußten eine verständliche Abneigung gegen den Militärdienst erwecken und damit das Aufkommen eines angemessenen Verhältnisses zur jungen nationalstaatlichen Gemeinschaft von vornherein verhindern. Das Überwiegen selbstsüchtiger Motive und persönlicher Ziele, der Verfall der Moral in der Verwaltung, die Untergrabung der Autorität und die Zerrüttung von Gehorsam und Disziplin im Heere mußten zu einer Verdrängung der konservierenden Faktoren und der ethischen Prinzipien und zu einer verhängnisvollen Entfaltung zerstörerischer Eigenschaften führen ${ }^{17}$.

Dieser Prozeß der Bildung anarchischer Zustände wurde für die jungen Republiken zu einer Belastung, die eine eigene Entwicklung im Keime ersticken

14 Wilhelm Hennis, Verfassung und Verfassungswirklichkeit. Tübingen 1968. - S. 12.

15 Hierzu F. Wehner, Der Konflikt zwischen spanischer und liberaler Staatsauffassung in Hispano-Amerika. In: Idee und Wirklichkeit in Iberoamerika, Beiträge zur Politik und Geistesgeschichte. Hamburg 1969, S. 25. Oder: VRƯ 1969, S. $41 \mathrm{ff}$.

16 Hierzu die Beiträge in Idee und Wirklichkeit.... vgl. Anm. 8), mit weiteren Literaturangaben, insbesondere zum Thema der Diktatur: G. Kahle, Historische Bedingtheiten der Diktatur in Lateinamerika. S. $55 \mathrm{ff}$.

17 G. Kahle, vgl. Anm. 11) - - für México dargestellt. 
mußte. Die Republiken übernahmen nicht eine in drei Jahrhunderten aufgebaute Autorität, nicht die führenden Kräfte aus Heer und Verwaltung. Offentliche Posten wurden oft zu Pfründen persönlicher Bereicherung und zur Ausübung persönlichen Ehrgeizes. Die Soldaten ebenso wie die Bevölkerung konnten kein Vertrauen zur Führung haben. Die Entwicklung war offen für Anarchie und Diktatur - die Demokratie als Institution versagte. „Los acontecimientos de la Tierra Firma nos han probado que las instituciones perfectamente representativas, no son adecuadas a nuestro carácter, costumbres y luces actuales ${ }^{18}$."

\section{Diktatur und Oligarchie}

Eine Untersuchung über die Herrschaftsformen und Lebensordnungen Spanischamerikas auf Grund ihrer geschichtlichen Herkunft wäre unvollkommen, wenn man nicht auch auf den Begriff der Oligarchie eingehen würde. Diese Bezeichnung wird in der politischen Polemik unserer Tage häufig angewandt als Kennzeichnung der prozentual kleinen Gruppe von Familien, die im Besitz des überwiegenden Anteils an Land, Wohlstand und Einfluß sind.

Nach der klassischen Definition des griechischen Philosophen Aristoteles ist Oligarchie die Herrschaft der Reichen zu ihrem Eigennutz. In einer vollen Oligarchie haben diese Reichen auch alle Regierungsämter inne. Aristoteles faßt die Oligarchie auf als Entartung der Aristokratie, der Regierung der Besten zum allgemeinen Wohle, wobei eine Anzahl gleich trefflicher Männer gemeinsam regiert.

Tatsächlich bildeten sich in Spanischamerika sehr rasch oligarchische Zustände. Für die Schaffung effektiver Grundlagen einer Demokratie wäre das Vorhandensein einer genügend stabilen mehrheitlichen Trägerschicht als repräsentativ für „das Volk" mit dem Willen zu ständiger Mitarbeit in der Gemeinschaft entscheidend gewesen. Hieran fehlte es; die einzelnen Volksgruppen gerieten nicht in einen Prozeß der Verschmelzung, sie standen sich eher feindlich, mindestens aber gleichgültig gegenüber.

Die treibenden Kräfte einer Erneuerung hätten in den Kreisen der nicht-landbesitzenden Spanischamerikaner der städtischen Intelligenz liegen können. Diese freisinnigen Kreise erwiesen sich aber als zu schwach, um eine staatstragende Rolle spielen zu können; eine für die Demokratie entscheidende bürgerliche Mittelklasse konnte sich damals noch nicht bilden. Vor allem fehlte es in diesen Teilen der Bevölkerung an klaren politischen Konzeptionen, an politischem Willen und Selbstbewußtsein. Soweit überhaupt von einem Mittelstand gesprochen werden kann, hatte sich dort eher eine gewisse Apathie und Abneigung gegenüber politischer Betätigung festgesetzt. So wollte der Mittelstand lange nicht, daß sich die Söhne im rauhen Klima des Parteilebens betätigten ${ }^{19}$, und es kam lange Zeit nicht zu einer Bildung politischer Traditionen in diesen Kreisen.

Auch die eigentlich zur politischen Führung berufenen Angehörigen der einflußreichen landbesitzenden kreolischen an Zahl kleinen Schicht erwiesen sich als politisch verhältnismäßig indifferent. Ihr Interesse lag an der Erhaltung ihres Besitzstandes, der Wahrung ihrer gesellschaftlichen Stellung und der auf den

18 Simón Bolívar, in der Carta de Jamaica, vgl. Anm. 9). - S. 34

19 Mauricio Solaún, El fracaso de la democracia en Cuba. - In: Aportes, Paris. Nr. 13/Julio 1969, S. 77. 
großen Gütern schon an Autonomie grenzenden Privilegien. Zwar waren die Aufstände hauptsächlich von diesen Kreisen getragen worden, doch zogen sie es nach Erlangung der staatlichen Unabhängigkeit vor, die Ausübung militärischer Dienste und der politischen Herrschaft dem Caudillotum zu überlassen, mit dem sich im Verein mit der Katholischen Kirche ein Zweckbündnis ergab, das das politische Leben in Spanischamerika verhängnisvoll belasten sollte und das die Einleitung einer für die Demokratisierung erforderlichen Integration aller Volksteile verhinderte.

Die vornehmlich von den Kreolen getragene Unabhängigkeitsbewegung war auch nicht von vornherein erstrebt worden mit dem Ziele der Einführung republikanisch-demokratischer Ordnungen. Vielmehr hatte der „wichtigste Grund in der spanischen Geschichte selbst" gelegen ${ }^{20}$. Die Unabhängigkeit hatte sich letzten Endes zwangsläufig ergeben aus dem Unverständnis der spanischen Krone gegenüber den amerikanischen Provinzen und ihren Bewohnern, die im Verlaufe von drei Jahrhunderten aus Spaniern zu Spanischamerikanern geworden waren. Die Lösung von der Krone hatte sich im Grunde mehr in einem Hin und Her der Gefühle und der Auseinandersetzungen gegen die lästige Bevormundung durch die spanischen Funktionäre vollzogen ${ }^{21}$. So ist z. B. in Nueva España, dem heutigen México, das Verlangen nach Unabhängigkeit durchaus nicht der Wunsch aller Bewohner gewesen ${ }^{22}$.

Die Bewohner von Spanischamerika waren in der Kolonialzeit auch kaum zu selbständigem politischem Handeln und Urteilen, ja nicht einmal zur Selbstverwaltung erzogen worden. Die Krone war stets darauf bedacht gewesen, die politische und verwaltungsmäßige Führung in der Hand zu behalten und der eingesessenen Bevölkerung keine Führungsrollen anzuvertrauen ${ }^{23}$. Die Bildung einer eigenen politischen Elite war damit systematisch verhindert worden durch planvolle Bewahrung des kolonialen Status. Diese Behandlung der Einwohner stand in krassem Gegensatz zu den Verhältnissen in Nordamerika, wo aus dem angelsächsischen Gesellschaftsdenken und dem Zwang zur Selbsthilfe der auf sich gestellten Siedler von Beginn an die Kunst einer eigenen politischen Gestaltung und Selbstverwaltung im demokratischen Sinne gedeihen konnte ${ }^{24}$.

Die Spanischamerikaner hatten es schwer, in dem kurzen Zeitabschnitt der Unabhängigkeitsbewegung eigene politische Ideen reifen zu lassen; es lag daher nur allzu nahe, sich an fremde Vorbilder zu halten. Eine Neigung zur Imitation wird sowieso dem Spanischamerikaner zugeschrieben: "Unfortunately the Latin American has always been an imitator in political and constitutional matters". Spanischamerika blieb geistig ,in the grasp of an inherited trinity of errors social medievalism, political and economic colonialism and reactionary clericalism“25. Die Unabhängigkeitsbewegung wurde nicht zu einer echten Revolution; der Geist der Kolonie mit dem Gegensatz zwischen den hierarchisch-autoritären und dirigistischen Tendenzen auf Grund der engen Verbindung zwischen Krone

20 Américo Castro, Spanien - Vision und Wirklichkeit. Köln/Berlin 1957. - S. 572. Weitere Belege auszugsweise in: Konetzke, vgl. Anm. 10), S. $44 \mathrm{ff}$.

21 Noch heute herrscht in Iberoamerika hierüber offenbar noch keine allgemeine Einsicht. Bezeichnend erscheint ein „Eingesandt" aus México in der Zeitschrift "Visión", 7. Nov. 1969, S. 6. - Der Einsender befaßt sich "mit den diktatorischen Militärregierungen in Paraguay, Bolivien, Argentinien und Brasilien und schließt: "Qué es lo que pasa en estos pueblos que tanto han luchado por su libertad? Serán necesarios nucvos héreos de la talla de San Martin, Bolívar, Morelos etc.?“

22 G. Kahle, vgl. Anm. 11), S. 245.

23 Siehe F. Wehner, vgl. Anm. 15), S. 32.

24 Hans Gustav Keller, Die Wurzeln der amerikanischen Demokratie. - Bern 1958. - S. 22 f.

$25 \mathrm{~J}$. Irizarry y Puente, The Nature and Powers of a "De Facto" Government in Latin America. - In: Tulane Law Review, Vol. XXX, 1955. - S. 15. 
und Kirche einerseits und der hemmungslosen individualistisch-egoistischen Freiheit der Besitzenden andererseits blieb erhalten. Gesellschaftsstruktur und koloniale Agrarwirtschaft blieben „básicamente intacta“26, abseits standen die Massen der meist analphabetischen Bevölkerung, die apathisch und resigniert ihr primitives Dasein weiterführen mußten. Gewisse sich aus der Ablösung der spanischen Bürokratie durch neue kreolische Kräfte ergebende soziale Umschichtungen $^{27}$ blieben vorläufig ohne politische Auswirkung auf eine Demokratisierung. -Die verkündete Gleichheit der Rassen war völlig illusorisch. Die Macht war in die Hände einer Koalition von Großgrundbesitzern, der hohen Geistlichkeit und gewissen Elementen des städtischen Bürgertums übergegangen, die ausreichende Unterstützung beim Militär gefunden hatte. Die Eingeborenen zogen keinerlei Nutzen aus dem Wandel der Lage“28. Den verantwortlichen Politikern hat lange die Vorstellung einer Verantwortung für die Integration der Eingeborenen gefehlt. Das neue in den Verfassungen festgelegte System blieb fremd. „Der Herr Präsident" blieb eine ebenso vage Vorstellung wie es vorher der König gewesen war. Unmittelbar vor dem Indio stand der "patrón“ oder der Dorfkacique mit dem ganzen Schwergewicht seiner autonomen Machtposition im Lebenskreis des Indios, der von jeher in obrigkeitlichem Geiste erzogen worden war. In der Weite des amerikanischen Raumes wurde die Ordnungsfunktion praktisch durch eine Vielzahl kleiner und größerer örtlicher und regionaler Diktatoren sui generis ausgeübt, die über Leben und Eigentum der Indios verfügten.

Die Art der spanischen Kolonialherrschaft hatte die Bildung einer echten Aristokratie aus der Erbschaft der Konquistadoren heraus unmöglich gemacht. Der von Natur aus aristokratischere Geist des spanischen „hidalgo“ erfuhr in Amerika eine Abwandlung zum Oligarchen. Daß keine Aristokratie entstanden war, hat auch Simón Bolívar erkannt ${ }^{29}$. Die zahlenmäßig geringe führende Schicht fällt infolge der Ausnutzung ihrer wirtschaftlichen und gesellschaftlichen Stellung in die Merkmale der Oligarchie. Auf die nach der klassischen Definition dazugehörige Ausübung der Regierungsgewalt hat sie weitgehend verzichtet zugunsten militärischer Caudillos und der direkten und indirekten Herrschaft, welche die Katholische Kirche ausübte. Die spanischamerikanischen Republiken wurden von einem diktatorisch-oligarchischen Mischsystem beherrscht, das durch eine formale, wenig mehr als nur in den Verfassungstexten existierende Demokratie getarnt war. Im Prinzip galt dies für alle Länder, wenn sich auch im einzelnen unterschiedliche Abstufungen entwickelten.

Aus dieser spezifischen Herrschafts- und Lebensform sind die Probleme dieser Staaten bis in unsere Tage hinein zu deuten. Wenn heute in der politischen Tagespolemik so häufig die Oligarchie verfemt wird, trifft das einen wesentlichen Teil des Systems, das die Bildung eines Volkes im Sinne einer ethnisch, sozial und kulturell homogenen Gruppe und damit die Grundlage für eine effektive Demokratie bis heute verhindert hat. Nur in wenigen Fällen, wie Uruguay, Costa Rica und auch Chile, waren die Voraussetzungen infolge der Zusammensetzung der Einwanderer im Zusammenhang mit der Ausrottung der Indios günstiger. In der überwiegenden Zahl der Länder bildeten sich heterogene Gruppierungen. Bei der Aufnahme des demokratischen Verfassungsmodells konnte das

\footnotetext{
26 Marcos Kaplan, vgl. Anm. 7). - S. 108.

27 Dargestellt von María del Carmen Velásquez: Nueva estructura social en Hispanoamérica. - In: Jahrbuch für Geschichte von Staat, Wirtschaft und Gesellschaft Lateinamerikas. Band 5/1958. - S. 264 ff. 28 Gustavo Beyhaut, Süd- und Mittelamerika II. - Fischers Weltgeschichte Band 23. 1965. - S. 30.

29 In einer Unterredung mit San Martín vom 26. 7. 1822. In: Konetzke, vgl. Anm. 10), S. $61 \mathrm{f}$.
} 
Grunderfordernis nicht erfüllt werden, wonach die Demokratie nur „für ein wahrhaft demokratisch denkendes Volk" eingeführt werden darf ${ }^{\mathbf{3 0}}$. In der Bevölkerung bildete sich nicht ein die Demokratie tragender spezifischer Typ des "common man"31 - ein geistig und sozial homogener Menschentyp mit ähnlichen Interessen.

\section{Demokratie und Liberalismus}

\section{Grundzüge des westlichen Liberalismus}

Zum Verständnis der Demokratie in Spanischamerika ist eine Gegenüberstellung des historischen Werdeganges der Demokratie in den "Modell-Ländern“ und ihrer Ideologien mit der hispanischen und spanischamerikanischen Tradition notwendig. Auf diesem Wege kann auch die Frage behandelt werden, von welcher Bedeutung die Ideologien der Aufklärung für die Erringung der Unabhängigkeit gewesen sind.

Für die Völker Westeuropas und die USA ist eine in den Grundzügen einheitliche geistige Ausrichtung dadurch gegeben, daß alle auf der Ideenwelt des politischen und ökonomischen Liberalismus basieren. Dieser hatte sich aus der Überwindung des Mittelalters aus Renaissance, Humanismus, Naturrecht und dem Kampf freiheitlicher Bürger gegen die Unfreiheit des Absolutismus entwickelt. Die westliche Demokratie beruht auf einer engen Liaison mit dem liberalen Gedankengut, Demokratie an sich braucht nicht immer damit verbunden zu sein. Die Begriffe Demokratie und Liberalismus sind daher prinzipiell nicht identisch; Demokratie kann in verschiedenen Formen und mit unterschiedlichem geistigen Inhalt auftreten ${ }^{32}$.

Ein Grundtyp und Träger des westlichen Liberalismus hatte sich schon früh in England seit der Revolution von 1689 durchgesetzt, in der Verbindung des ländlichen Kleinadels mit dem städtischen Kapital; Grundbesitz und kaufmännischer Geist hatten sich vereint. Dieser ursprüngliche englische Liberalismus war weder demokratisch, noch egalitär, noch aggressiv rationalistisch oder antireligiös, wie der spätere kontinental-europäische ${ }^{\mathbf{3}}$.

Eine demokratische Ausprägung fand die freiheitliche Bewegung in den angelsächsisch kolonisierten und eroberten Gebieten Nordamerikas. Von Beginn an bestand eine innere Übereinstimmung zwischen den materiellen Bedürfnissen des Siedlers, dem politischen Willen zur demokratischen Ordnung der Gemeinschaft und dem religiösen Impetus. Die aus England ausgewanderten Siedler und Unternehmer wollten sich selbst regieren, sie mußten im Kampfe um die Selbsterhaltung zusammenstehen, und sie wollten ihr politisches Dasein nach eigenen Ideen und Erfordernissen ausrichten. Mit ausschlaggebend für eine eigene schöpferische Gestaltung war im Gegensatz zu Spanischamerika das Fehlen feudaler Schichten. Vom Ursprung an waren hier "experience and background for democratic government" gegeben, die in der Verfassung von 1787 ihren Niederschlag fanden. Sie zeichnet sich aus durch eine typische Regelung des Verhältnisses des Volkes

30 Carl Schmitt, Die geistesgeschichtliche Lage des modernen Parlamentarismus. 2. Aufl., München 1926. S. 36.

31 C. J. Friedrich, vgl. Anm. 4), S. $39 \mathrm{ff} ., 109 \mathrm{ff}$.

32 id. S. 83 ("Antithese von Demokratie und Liberalisierung“). Carl Schmitt, vgl. Anm. 30), S. 30.

33 Friedrich A. v. Hajek, Politischer Liberalismus. In: Handwörterbuch der Sozialwissenschaften, 6. Band, S. 592. - Göttingen 1959. 
zur Regierung mittels Begrenzung der Herrschaft durch Trennung der drei Gewalten Legislative, Exekutive und Justiz. Der Präsident hat zwar starke Kompetenzen, ist aber durch unabänderliche Garantierung der hauptsächlichen individuellen Grundrechte, die auch der Kongreß nicht zu ändern vermag, sowie durch vorrangige Befugnisse des Kongresses in der Bewilligung finanzieller Mittel gebunden. In der hohen Bedeutung des Parlamentes als der Stätte öffentlicher Diskussion prägt sich der Geist des westlichen Liberalismus deutlich aus. Das gilt auch für den rationalen Gehalt dieses Systems, das im Geiste des 18. Jahrhunderts konzipiert war. Demokratie muß danach eine „freie, nach Spielregeln rational kontrollierte Zusammenarbeit von Gleichberechtigten sein"34. Im Rahmen dieses Verhältnisses bleibt dem einzelnen viel Spielraum für politische Mitwirkung und wirtschaftliche Initiative und Bewegungsfreiheit. Eine spezifische Bedeutung kommt der Verfassung zu, die zur "politischen Bibel“ des nordamerikanischen Volkes wurde, zum „Gefäß und Inbegriff der Werte, die diesen Staat gebildet und geformt haben und ihn erhalten" 35 .

Ihre Stärke besonderer Art zieht die nordamerikanische Demokratie aus dem Geist des Puritanismus, der ihr ihren Schwung, das Gefühl der religiösen Rechtfertigung und zugleich ein Empfinden für moralische Verantwortlichkeit des Bürgers verleiht ${ }^{36}$. Puritanismus und Demokratie kann man als gleichrangige Wurzeln der nordamerikanischen Ideologie ansehen.

Der Puritanismus gibt der Demokratie ein missionarisches Sendungsbewußtsein und erzeugt einen Bekehrungseifer aus dem Glauben an die universelle Geltung dieser Demokratie. Wer für die so verstandene Freiheit und gegen den autoritären Staat als unchristliche Tyrannei schlechthin ficht, entspricht dem Willen Gottes $^{37}$. Die Auserwähltheit wird zugleich im irdischen Erfolg sichtbar; hier liegt die entscheidende Wurzel für den nordamerikanischen Aktivismus. Zugleich wird hier der Gegensatz zu dem Lateinamerikaner sichtbar. Dem Nordamerikaner bleibt die so ganz andere geistige Einstellung und historische Entwicklung Lateinamerikas aus seiner meist einseitig puritanisch-demokratischen Haltung heraus häufig fremd; er sieht vieles oberflächlich und verzerrt. Der Lateinamerikaner fühlt sich in seiner Wirklichkeit verkannt. In diesem religiösweltanschaulichen Gegensatz, dem beiderseitigen Unverständnis und der daraus folgenden Politik der USA liegt letzten Endes die Begründung für das unbefriedigende und gestörte Verhältnis zwïschen den beiden Teilen Amerikas.

Besonders in der Geschichte und Art der Kolonisierung und Eroberung Spanischamerikas liegen so starke Unterschiede, daß eine grundsätzliche Vergleichbarkeit nicht gegeben ist. In Spanischamerika geschahen Eroberung und Besiedlung aus anderen Motiven als in Nordamerika. Der Spanier war ein Konquistador, ein Eroberer, der aus einer katholischen Kreuzzugsidee heraus neue Provinzen für die spanische Krone in deren Auftrage und damit zugleich neue Seelen für seinen Glauben gewinnen wollte. Ihm lag nicht das Siedler- und Unternehmerideal angelsächsischer Prägung - er war Krieger und suchte das sagenhafte Goldland. Er brachte keine irgendwie geartete demokratische Vorstellung oder Tradition mit, sein Denken und Handeln spielte sich im geistigen Bereich der traditionel-

\footnotetext{
34 Golo Mann, Vom Geist Amerikas. 2. Aufl. - Stuttgart 1955. - S. 98.

35 H. G. Keller, vgl. Anm. 24). - S. 51.

36 Knud Krakau, Missionsbewußtsein und Völkerrechtsdoktrin in den Vereinigten Staaten von Amerika. -

Frankfurt 1967.
S. a. H. G. Keller, vgl. Anm. 24). - S. $29 \mathrm{ff}$.

37 Gerhard Ritter, Das deutsche Problem. - München 1962. S. 14.
} 
len spanischen Hierarchie ab. Der Spanier ist seiner geschichtlichen Natur nach ein "hidalgo", ein Individualist, der nicht das gleiche Verhältnis zu Arbeit und westeuropäischer Nützlichkeit hat wie etwa der US-Bürger ${ }^{38}$. In Altspanien hatten Fleiß und gewerbliche Tüchtigkeit den Mauren, den „Ungläubigen“ obgelegen. Nur in wenigen Ausnahmefällen (vor allem in Uruguay und Costa Rica) setzte sich die Einwandererschicht aus mittelständischen Bürgern, Siedlern, Handwerkern und Kaufleuten zusammen als natürliche Basis für eine demokratische Ordnung. Der Ecuadorianer und derzeitige Generalsekretär der Organisation der Amerikanischen Staaten (OAS), Galo Plaza Lasso, hat die Ansicht vertreten, daß zwischen den USA und Lateinamerika der gleiche historische „background“ bestehe; "the people in Latinamerica are basically democratic"39. Dieser Ansicht kann nicht zugestimmt werden, alle grundsätzlichen Verschiedenheiten sprechen dagegen. Die Nordamerikaner sind geborene Demokraten, die Spanischamerikaner sind es nicht.

Für den politischen wie auch für den wirtschaftlichen Liberalismus gilt das Gesetz der ungehinderten Betätigungsmöglichkeit der individuellen Initiative und der Ausbalancierung dieser Kräfte in freier Konkurrenz. Der verbindliche Wille muß in einem Prozeß ständiger unbehinderter Diskussion und Konkurrenz zwecks Überwindung von Irrtümern, Ermittlung der Wahrheit und Erzielung des besten Ergebnisses immer von neuem gesucht und formuliert werden. Wenn der einzelne seine privaten und persönlichen Interessen und Meinungen aktiv verfolgt und vertritt, so dient er damit im Gesamtprozeß „naturgesetzmäßig“ der Erzeugung der Wohlfahrt des Ganzen und der Wahrheit. Eine spezifische Ausprägung und Anwendung fanden diese Ideen in der klassischen Nationalökonomie. Die Verfolgung widerstreitender ökonomischer Interessen in freiem Wettbewerb gewährleistet die "prästabilisierte Harmonie“. Voraussetzungen sind vor allem die Befreiung des einzelnen aus den Bindungen des Absolutismus und der Standesgliederung sowie gleiche Startbedingungen. Die freie Wettbewerbswirtschaft ist geradezu das Herzstück des westlichen Liberalismus.

In Spanischamerika hat eine solche Grundlage des politischen wie auch des wirtschaftlichen Liberalismus während der Bildung der selbständigen Staaten weder theoretisch noch praktisch Eingang finden können. In den Verfassungen wurden zwar das private Eigentum und andere Grundrechte garantiert - in der Praxis wurde kaum davon Notiz genommen. Spanischamerika hat eine imperfekte Wirtschaftsstruktur geerbt, wie sie in der Kolonialzeit aus merkantilistischen und kolonialen Überlegungen gestaltet worden war - dieses Erbe wurde aber auch im alten Sinne konserviert. Die Spanischamerikaner haben kein Verhältnis zur liberalen Wirtschaftsform des freien Wettbewerbs finden können; mehr noch als der politische blieb der ökonomische Liberalismus fremd. Als Sinn und Zwang der spanischen Kolonialregierung entfielen, blieb eine feudale kapitalistische Struktur, in der es kaum noch eine Chance eines sozialen Schutzes für die Masse des Volkes gab, aber auch nicht die Möglichkeit einer Mitwirkung und Initiative fast aller Kreise außerhalb der Nutznießer des Systems, vor allem der oligarchischen Oberschicht. Die Wirtschaft verharrte in der semikolonialen und monokulturellen Form und blieb im modernen Sinne steril - ein Ergebnis, das weder mit Demokratie noch speziell mit Liberalismus etwas zu tun hat. Die spanisch-

38 Hierzu: Fernando Guillén Martínez, Raiz y Futuro de la Revolución. Bogotá 1963, S. 36 ff.

39 Golo Plaza Lasso, Problems of Democracy in Latin America. - The University of North Carolina Press, 1955. - S. 43. 
amerikanischen Länder traten den Marsch in das 19. Jahrhundert mit eigenem, jedenfalls undemokratischem und unliberalem Gepäck an, das auch im 20. Jahrhundert noch den Weg zum Fortschritt ungebührlich behindern mußte.

\section{Aufklärung und Kirche}

Trotz der Abschließung der kolonialen Provinzen und trotz scharfer Zensur war Schrifttum der Aufklärung auch schon vor der Emanzipation in die spanischamerikanischen Länder auf vielfachen Wegen eingedrungen ${ }^{40}$. Das gilt allerdings vornehmlich für politische Autoren wie Voltaire, Montesquieu, Rousseau u. a. sowie für Philosophen des 17. und 18. Jahrhunderts. Schrifttum aus dem Bereich des wirtschaftlichen Liberalismus wird in den Berichten aus der damaligen Zeit kaum erwähnt; es fand offenbar keinen Anklang41. Aber nicht nur für das wirtschaftliche, auch für das politische Schrifttum bleibt die Frage nach der Tiefenwirkung. Zwar eignete man sich das Vokabular der Aufklärung an und berief sich zur Begründung des Aufstandes auf die „natürlichen“ Rechte gegen die „Tyrannen ". Insgesamt darf aber wohl der Einfluß der außerspanischen geistigen Einwirkung nicht überschätzt werden - klare Konzeptionen sind offenbar daraus nicht entstanden, die neuen Ideen haben in den Köpfen eher mehr wirre als klare Ideen entstehen lassen ${ }^{42}$. Die philosophisch-politische Aufklärung hat in Spanischamerika geringeren Einfluß ausgeübt als im Mutterlande selbst. Für die Erringung der Unabhängigkeit war letzten Endes der Bruch des traditionellen Verhältnisses zur Krone entscheidend; gegenüber den europäischen Ideen der Aufklärung entstand eher noch eine gewisse Opposition ${ }^{43}$.

Es regten sich vor allem religiöse Gefühle, die sich gegen den antiklerikalen „französischen Unglauben“ und die radikal demokratischen Ideen wandten ${ }^{44}$. Der Einfluß der katholischen Kirche blieb vorherrschend. Hier zeigte sich die tiefe Verwurzelung des Katholizismus aus der Kreuzugsidee heraus, unter der die Konquistadoren $\mathrm{zu}$ ihrem Eroberungszuge angetreten waren. In vielen Zeugnissen aus den Jahren der Unabhängigkeit kommt die unerschütterliche Verpflichtung zum Ausdruck, "die heilige Religion zu bewahren" ${ }^{45}$; sie wurde auch institutionell verankert. In der Verfassung von Cádiz von 1812 heißt es in Art. 12: „La religión de la Nación Española es y será perpetuamente la católica, apostólica, romana, única verdadera. La Nación la protege por leyes sabias y justas y prohibe el ejercicio de cualquier otra" (siehe dazu auch Art. 173). - Ähnlich formuliert es die Verfassung von Perú von 1823, in den Art. 8 und 9 - um nur einige Beispiele zu nennen. - Ein seltenes Gegenbeispiel aus jener Zeit bietet der paraguayische Diktator Dr. Francia, der eine christliche, vom Klerikalismus befreite Republik errichtete und eine paraguayische Nationalkirche gründete ${ }^{46}$. Wie auch immer im

40 Arthur P. Whitacker (ed.), Latin America and the Enlightenment - 2. Aufl., New York 1958. S. 12 u. a.

41 Roland D. Hussey, in der Sammlung Whitacker, vgl. Anm. 40), - Traces of French Enlightenment in Colonial Hispanic America. - S. 40 .

42 G. Kahle, vgl. Anm. 11). S. 186 f., 192. García Calderón, vgl. Anm. 6), S. 173.

43 John Tate Lanning, in der Sammlung Whitacker, vgl. Anm. 40). - The Reception of the Enlightenment in Latin America. - S. $85 \mathrm{ff}$

O. Carlos Stoetzer, Die geistigen Grundlagen der spanisch- amerikanischen Unabhängigkeit. - In: Idee und Wirklichkeit in Iberoamerika, vgl. Anm. 8). - S. $81 \mathrm{ff}$.

44 Charles C. Griffin, The Englightenment an Latin American Independence. - In Whitacker, vgl. Anm.

45 Iturbide in der Proklamation von Iguala, 24. 11. 1821. In: R. Konetzke. Vgl. Anm. 10). S. 50.

46 Günter Kahle, Die Diktatur Dr. Francias. - In: Jahrbuch für Geschichte von Staat, Wirtschaft und Gesellschaft Lateinamerikas. - Band 1/1964. - S. $259 \mathrm{f}$. 
einzelnen die formell staatsrechtliche Regelung in den jungen Staaten erfolgte, der geistig überragende und im Kern intolerante Einfluß der katholischen Kirche blieb erhalten, wenn auch eine von Wechselfällen beherrschte Emanzipierung schon im Laufe des 19. Jahrhunderts einsetzte.

Der politische Glaube Spanischamerikas blieb die scholastische Idee, wonach der Mensch im Dienste Gottes stehe. Die katholische Religion wurde als verfassungsmäßige Institution und als Grundlage der geistigen Einstellung auch von den Republiken übernommen. Deren Ideen stehen denjenigen des Liberalismus mit seiner Voranstellung des Menschen und seines irdischen Glücks diametral entgegen. Eine religiöse Intoleranz ist kein Boden für die Entfaltung liberaler Meinungsfreiheit. Im Gegensatz dazu heißt es in der Präambel der USA-Verfassung, daß diese die Wohltat der Freiheit gewähren soll und in Art. I der 1. Verfassungsänderung, daß der Kongreß kein Gesetz zur Etablierung irgendeiner Religion erlassen und die freie Ausübung nicht verbieten darf.

Vor allem die enge und einseitige Bindung an die katholische Religion hat den geistigen Übergang Spanischamerikas aus mittelalterlich-scholastischen Vorstellungen in die neue Zeit ungebührlich erschwert. Der Traditionalismus wurzelt im Katholizismus, in der "Wesenseinheit von Hispanidad und Katholizismus" ${ }^{47}$. Die katholische Kirche hat in ihrem engen Bündnis mit Oligarchie und Diktatur den blinden Glauben an die Obrigkeit konserviert und dazu beigetragen, daß die rückständige Sozialstruktur bewahrt und der Weg in eine demokratische Entwicklung versperrt wurde. Sie ist es vor allem, die den Spanischamerikaner immun machte gegen die Ideen der Aufklärung, die die geistige Auseinandersetzung mit den Ideen des 17. und 18. Jahrhunderts gehemmt hat und den Weg Spanischamerikas in die Neuzeit noch heute so viel beschwerlicher sein läßt als irgendwo sonst. Spanischamerika ist - ähnlich wie Spanien, dem Umbruch aus dem Mittelalter in die Neuzeit aus dem Wege gegangen. Für den hispanischen Konservatismus gilt immer noch der Satz: "La religión católica es el mas fecundo elemento de regeneración que se abriga en el seno de la nación española“48. Die "unidad religiosa" verleiht den inneren Halt gegenüber den Wirren der Welt. Hier liegt die eigentliche Affinität zum Katholizismus und das Mißtrauen gegenüber einer rationalisierten entzauberten Welt des Liberalismus - ein unversöhnlich erscheinender Gegensat ${ }^{49}$.

Die spanischamerikanische Seele hat die Herausforderung des Liberalismus nicht angenommen, sie ist "in sich selbst verharrt geblieben"50, sie hat sich gegen den Prozeß der inneren Säkularisierung gestemmt und sich nach außen abgeschlossen.

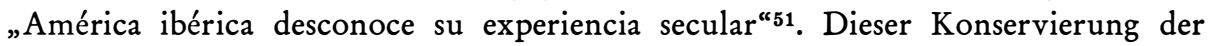
hispanischen Substanz entspricht die Eigenschaft, Masken aufzusetzen, ohne den Wesenskern $\mathrm{zu}$ verändern ${ }^{52}$. Der Spanischamerikaner entwickelt dabei eine Eigenschaft, die als "mimetismo“ bezeichnet worden ist ${ }^{53}$, wobei dahingestellt bleiben mag, ob dieser Ausdruck das spanischamerikanische Phänomen voll kennzeichnet. Die Ideen der Aufklärung wurden nicht zu einer verbindlichen Philosophie rezi-

47 Joachim Fernandez, Spanisches Erbe und Revolution. - Münster 1957. - S. 111 f.

48 Jaime Balmes, En Busca del Remedio. - Auszugsweise in: "España como Preocupación", Hrsg. v. Dolores Franco. Ediciones Guadarrama, Madrid 1960, 2. Aufl. - S. 210.

49 J. Fernandez, vgl. Anm. 47).

50 Américo Castro, vgl. Anm. 20), S. 286.

51 C. Pisano Fischer, vgl. Anm. 1). - S. 129.

52 Fernando Guillén Martínez, Los Estados Unidos y América Latina. In: Aportes, Paris, Nr. 7/Enero 1968. - S. 11 .

53 id., S. 12. 
piert; der spanische Geist weiß fremde Ideen nur insoweit zu verarbeiten, als sie seiner traditionellen Geistesart entsprechen. Andererseits wurde der äußeren Form durch Aufnahme liberal-demokratischer Verfassungen Genüge getan. -

Man kann die Wirkung fremder geistiger Einflüsse auf Philosophie und Staatsdenken auch an den Einwirkungen französischen Geistesgutes abmessen. Man sprach zeitweise geradezu von einer „época franciana“, in der die allgemeine Neigung des Spanischamerikaners zur Bewunderung ausländischen Geistesgutes besonders hervortrat. Dieser französische Einfluß findet seinen Niederschlag in dem 1911 geschriebenen Buch von F. García Calderón ${ }^{54}$, in dem die "große lateinische Familie" verherrlicht wird: „Das ursprünglich spanische und portugiesische Amerika wird schließlich kulturell französisch". Das Buch erschien mit einer Widmung von Raymond Poincaré. Heute könnte eine solche Auffassung gegenüber der tatsächlichen Entwicklung nicht mehr aufrechtzuerhalten sein. García Calderón war der durch die Entwicklung nicht bestätigten Ansicht, daß „einige iberoamerikanische Völker ihr klägliches Erbteil abgeschüttelt haben. Auch jenseits des Meeres werden Freiheit und Demokratie schrittweise verwirklicht" $" 55$. Gegenüber den liberalen, und damit auch gegenüber den französischen Ideen hat schließlich doch das „Bollwerk der scholastischen Theologie" standgehalten ${ }^{56}$. Die fremden Einflüsse haben nur die Oberfläche berührt, eine innere Rezeption hat nicht stattgefunden.

Gegenüber den vielen äußerlich gebliebenen Einflüssen in Verbindung mit der inneren Verkettung an den intoleranten Katholizismus haben sich eine eigene Philosophie, eigenes geistiges Selbstbewußtsein und eigene Staatsideen während des Prozesses der Erlangung der Unabhängigkeit nicht bilden können. Bis weit in das 20. Jahrhundert hinein ist eine ideologische Schwäche geblieben, ein eigenes „pensamiento político" hat sich unter der Maske der fremden Einflüsse nicht entwickeln können. Gegenüber der aggressiven Philosophie des Liberalismus beginnt sich erst neuerdings eine ebenbürtige philosophische Position herauszukristallisieren ${ }^{57}$. Sicher hängt diese Tatsache auch mit dem Menschentyp zusammen; erst wenn sich die Rassenmischung vollendet zu einer allgemeinen „mestizaje“, wird daraus auch eine Philosophie dieses Menschentyps erwachsen. Das bisher vorwiegend im Wunschdenken vorhandene eigene „sehr histórico“ kann dann politische Wirklichkeit werden.

Aus der katholischen Grundlage heraus erklärt sich auch die spanischamerikanische Neigung zu apokalyptischen Visionen, mit denen man die unvollkommene, ungewünschte irdische Realität zu überspielen sucht ${ }^{58}$. Während der Liberalismus die Welt perfektionieren will, sucht sich der Katholik von der Welt zu befreien, er sucht „totale“ Lösungen magischer Art. Hier liegen die Wurzeln der Neigung zu messianischen Träumen und zum charismatischen Führertum. Durch die Geschichte geht der gleiche Zug, ob die Azteken auf die Rückkehr Quetzalcoatls warten oder ob der Indio dem Wunderglauben der katholischen Kirche verfällt. Auch die für Außenstehende auffällige Glorifizierung der Freiheitshelden gehört ebenso hierher wie die charismatisch-messianische Führerrolle Fidel Castros, die als „altamente

54 Vgl. Anm. 6).

55 id. S. 2 .

56 Jaime Eyzaguirre, Der Abschluß des 19. Jahrhunderts in Chile. - In: Aconcagua, Vol. 5/Nr. 1/1969. S. 32 .

57 Siehe den Überblick über diese Entwicklung in: Leopoldo Zea, Philosophy and Thought in Latin America. - In: Latin American Research Review, III/2, 1968. S. 3 ff.

58 M. Solaún, vgl. Anm. 19), S. 71 (mit Zitierung von Albert O. Hirschman). 
milenario-mesiánico y no secular" bezeichnet worden ist ${ }^{59}$. Die spanische Expansion entsprang nicht primär aus Habsucht und Grausamkeit, sondern aus Träumen aller $\mathrm{Art}^{60}$. Es bildete sich eine pseudo-política mitología"61.

In der Wirklichkeit westlicher Liberal-Demokratien hat die Entwicklung zwar auch nicht zu einer totalen Säkularisierung geführt. So gilt in England immer noch der aus der Frühzeit stammende Satz, wonach die Demokratie ein „religious and moral principle" sei ${ }^{62}$. Verbindungen und Abgrenzungen der Anglikanischen Kirche zu Staat und konstitutioneller Monarchie sind intakt geblieben und damit auch die religiöse Legitimierung von Staatsform und Autorität. - In den USA hat der religiöse Geist des Puritanismus von Beginn an die Demokratie erfüllt und geprägt; bei scharfer institutioneller Trennung besteht doch eine enge Verbindung zwischen dem religiösen und dem politischen Denken. Die US-Demokratie ist auch heute noch die "stärkste, radikalste Demokratie"63, sie ist doktrinär, ihre Anziehungskraft liegt in dem Selbstbewußtsein, mit dem sie sich anbietet. Das entscheidende Merkmal ist die innere Einheit zwischen Glauben und politischer Form, woran es in Spanischamerika fehlt, nachdem eine solche Einheit von Form und Inhalt sich schon im Ansatz nicht gebildet hatte. Im Gegenteil ist in diesem Sinne die demokratische Idee nicht rezipiert worden; die Demokratie blieb ein Lippenbekenntnis in den Verfassungstexten, und der Liberalismus stand im Gegensatz zu dem religiös verhafteten Traditionalismus, wenn auch vielleicht nicht in so scharfer Form wie in Spanien.

\section{Demokratie und spanischamerikanischer Liberalismus}

\section{Wandlungen der jüngsten Zeit}

Gegenüber dem bisher ermittelten Tatbestand eines im wesentlichen mit obligarchisch-diktatorischem Geiste erfüllten republikanischen Systems ist der Eindruck nicht wegzuleugnen, daß die Durchsetzung wirklicher Demokratie in Spanischamerika voller Probleme steckt. Nun macht sich aber in den allerletzten Jahrzehnten ein deutlicher Wandel bemerkbar. Die traditionelle oligarchisch-starre und ademokratische Gesellschaftsstruktur beginnt sich aufzulockern, so daß die Voraussetzungen für einen Demokratisierungsprozeß entstehen. Gewisse Vorläufer sind Bewegungen wie der Aprismus in Perú oder soziale Tendenzen in der mexikanischen Verfassung von 1917, um nur einige Beispiele zu nennen. Diese Bewegungen blieben aber auf das jeweilige Land begrenzt. Eine über die Ländergrenzen hinweg ganz Spanischamerika ergreifende Wandlung beginnt erst nach dem Zweiten Weltkrieg. Sie setzt realistisch ein mit dem durch die Tätigkeit der Comisión Económica para América Latina (CEPAL) gekennzeichneten Bemühen um eine gemeinsame Wirtschafts- und Integrationspolitik. Sie setzt sich fort damit, daß die geistigen Anseinandersetzungen auf weitere Bereiche übergreifen. Auch das Thema der "Demokratie“ dringt in das öffentliche Bewußtsein und wird ein Gegenstand

\footnotetext{
59 id. S. 73, Anm. 86. Siehe auch: Virginia Prewett, A Changing United States policy. - In: The Caribbean, I/Vol. XVI. - Gainsville 1966. - S. 218.

60 Américo Castro, vgl. Anm. 20), S. 572.

61 F. Guillén Martínez, vgl. Anm. 52), S, 12.

62 Zitiert in: Gerhard Leibholz, Das Wesen der Repräsentation und der Gestaltwandel der Demokratie im 20. Jahrhundert. 3. Aufl., Berlin 1966. S. 211.

63 Golo Mann, vgl. Anm. 34), S. 140.
} 
ernsthafter Diskussionen und praktischer politischer Zielsetzungen, nicht zuletzt auch von Militärregierungen. Mag heute der Erneuerungsprozeß noch vielfach unklar erscheinen - der Trend zu einer Úberwindung der „obstáculos“, der hemmenden Faktoren durch zeitgemäße Reformen ist unverkennbar.

Jetzt wird man sich bewußt, daß man in der Konfrontierung mit den Erfordernissen der modernen industriellen Gesellschaft vor Problemen steht, die mit den traditionellen Methoden und Auffassungen nicht mehr zu lösen sind. Die durch die Industrialisierung einsetzende Diversifizierung der Wirtschaft, die Überwindung der semikolonialen Strukturen und der einseitigen feudalen Agrarwirtschaft mit dem Ziele einer gerechteren Einkommensverteilung und Beteiligung aller Volksschichten an der nationalen Arbeit bewirken zugleich eine soziale Umschichtung und erfordern die Beseitigung überkommener sozialer Diffamierungen. Moderne Verkehrs- und Kommunikationsmittel lösen traditionelle Gebundenheiten auf. Es wächst ein stärkerer Mittelstand als künftige politische Trägerschicht heran. Die Verantwortung für die in der Marginalität lebenden unteren Schichten ist erwacht; Alphabetisierung und Reform der Daseinsbedingungen werden vordringliche Ziele. Die Unzufriedenheit mit dem bisherigen System macht sich überall geltend - die oligarchische Hegemonie bröckelt ab, denn sie ist überholt und nicht mehr zu konservieren; auch nicht in denjenigen Ländern, die noch nicht wie z. B. México oder gar Cuba auf revolutionären Wegen mit alten Vorstellungen aufgeräumt haben. - Die neuen Auffassungen schlagen sich in Verfassungsänderungen nieder, in denen z. B. der liberale Begriff des privaten Eigentums abgelöst wird durch Auffassungen sozialer Verpflichtung des Eigentums ${ }^{64}$.

\section{Grundmerkmale der Demokratie}

Solche Anzeichen des Aufkommens eines neuen und moderneren Spanischamerika legen die Frage nahe, ob und inwieweit die bisher verwandten Begriffe überholungsbedürftig sind. Ist es richtig, den Begriff „Demokratie“ unverändert weiterhin auf Spanischamerika anzuwenden? Muß nicht insbesondere der Wertgehalt, den die moderne Demokratie durch den Liberalismus erhielt, auf seine Brauchbarkeit für Spanischamerika überprüft werden? Das Problem liegt darin, daß die so auffällige Diskrepanz zwischen imitierten Modellen und politischer Wirklichkeit darauf zurückzuführen ist, daß sich eine Demokratie nur dann nicht mit dem Charakter Spanischamerikas verträgt, wenn sie vom Geist des westlichen Liberalismus erfüllt ist.

Man wird, anders als es bisher generell geschehen ist, die Begriffe Demokratie und Liberalismus auseinanderzuhalten haben, um für Spanischamerika zu klaren Vorstellungen zu gelangen. Man wird sich dabei an den Grundmerkmalen der Demokratie orientieren müssen, um die Anwendbarkeit auf Spanischamerika zu klären.

Der Grundbegriff jeder Demokratie, abstrahiert von den Wertvorstellungen des westlichen Liberalismus, beruht auf der wörtlichen Bedeutung, die dem griechischen Ursprung nach "Volks"-(demos),Herrschaft"(kratein) ist. Demokratie ist eine "Herrschafts- und Lebensform "65. Nach der aristotelischen Definition ist Demokratie die Herrschaft der Armen zu ihrem Vorteil; sie sind die Mehrheit; das Merkmal ist die Gleichheit der Freien.

64 José Miranda, Reformas y Tendencias Constitucionales Recientes de la América Latina. - Mérico 1957. 65 Vgl. Anm. 4). 
Alle Gewalt geht vom Volke aus und nicht von einer Obrigkeit von höheren Gnaden; sie wird zum Wohle des Volkes ausgeübt. Nach einem Wort von Lincoln soll es sein ein "government of the people, by the people and for the people". Das Volk will und muß durch periodisch wiederkehrende Wahlen selbst bestimmen, wer Repräsentanz und Herrschaft in seinem Namen ausüben soll, es will darüber hinaus durch öffentliche Meinungsbildung ständigen Einfluß auf die Regierungstätigkeit nehmen, die keine geheime Kabinettspolitik sein darf. Zwischen dem Volk und der Regierung muß ebenso wie unter den Bürgern selbst Übereinstimmung über die Grundlagen und Verhaltensweisen herrschen („agreement on fundamentals") ${ }^{66}$.

Entscheidend für die Funktionsfähigkeit ist ein ausgewogenes Verhältnis zwischen dem individuellen Begehren und dem $\mathrm{Maß}$ an Herrschaftsbefugnissen, das der Regierung zur Verfügung gestellt (gestattet) wird, d. h. die „Wechselbeziehung zwischen Führer und Geführten" ${ }^{\text {67 }}$.

Das Volk muß diese Art von Herrschaftsübertragung ebenso wie ein mögliches Maß an individueller Freiheit sowie politische, rechtliche und gesellschaftliche Gleichheit w olle n. Demokratie selbst wie auch Freiheit und Gleichheit sind keine abstrakt gültigen Begriffe, ihr Gehalt wird durch die Wertvorstellungen des betreffenden Volkes, seine Geschichte und Umgebung bestimmt. Die verschiedenen Völker oder sozialen und ökonomischen Gruppen, die sich „demokratisch“ organisieren, haben nur abstrakt dasselbe Subjekt "Volk“. In concreto sind die Massen soziologisch und psychologisch heterogen "68. Demokratie tritt in unterschiedlichen Anwendungen und Formen auf, in politisch abweichenden und gegensätzlichen Systemen. Zwischen einer überwiegend parlamentarischen Form einerseits und der Bezeichnung totalitär-diktatorischer Regime als „Volksdemokratien“ andererseits liegt ein weiter Abstand.

Es gibt daher keine allgemein gültige Auslegung des Begriffs Demokratie; selbst in den USA, das als klassisches Land der modernen Demokratie bezeichnet werden kann, hat sich eine eindeutige Begriffsbestimmung nicht gebildet ${ }^{69}$.

Wenn auch Demokratie und Liberalismus im Grundsatz begrifflich auseinanderzuhalten sind, so stehen sie dennoch in einem verwandtschaftlichen Verhältnis zueinander. Jede echte Demokratie bedarf eines freisinnigen, d. h. „liberalen“ Geistes. Für eine spanischamerikanische Demokratie erhebt sich daher die Frage, welche Grundelemente der Demokratie und welche Spielart von Liberalismus ihr Inhalt, Wert und Funktionsfähigkeit zu verleihen vermag. Dies gilt für die Grunderfordernisse Freiheit und Gleichheit ebenso wie für die Regelung der Herrschaftsverhältnisse.

\section{Freiheit und Gleichheit}

Die Problematik einer spanischamerikanischen Demokratie beginnt an der Grundfrage nach dem Volke, das als Substanz existieren und die demokratische Form auf der Grundlage von Freiheit und Gleichheit wollen, d.h. einen eigenen politischen Willen zum Ausdruck bringen muß. Nachdem in der Kolonialepoche

68 Carl Schmitt, vgl. Anm. 30), S. 34. S. a. Gerhard Leibholz, vgl. Anm. 62). S. 46 ff.

69 H. G. Keller, vgl. Anm. 24) - S. 11 und 54 (Anm. 18). 
keine Bildung homogener Völker stattgefunden hatte und auch im Verlaufe des Prozesses der Unabhängigkeit überwiegend kein Volk in demokratischem Sinne entstehen konnte, steht dieses Problem auch heute noch vor der Lösung, die sich allerdings andeutet in dem Entstehen eines betonten Nationalismus. Gerhard Masur hat dessen Entwicklung das wichtigste Phänomen in Lateinamerika genannt. „. . only through the adherence to the tenets of Mexicanism, Argentinidad, Brazilian destiny, Chileanism etc. could these countries have hoped to overcome the handicaps of semicolonialism that had delayed their evolution in the contemporary world." "Democratic government may not necessarily be the answer to the problems, but popular awakening and popular participation are indispensible if they are to be rescued from the doldrums of 150 years of public apathy "70.

In der Ideologie des westlichen Liberalismus nehmen Freiheit und Gleichheit einen hervorragenden Platz ein. Es ist das erklärte Ziel des aufgeklärten Bürgertums, sich seine Ansprüche auf einen individuellen Betätigungsraum als "Grundrechte“ verfassungsmäßig garantieren zu lassen; das ideologische Schwergewicht liegt bei diesem Begehren.

Wenn auch die Formulierungen in den Verfassungen der spanischamerikanischen Länder ebenfalls die Grundrechte garantieren, so doch nicht in der Unabdingbarkeit wie nach der USA-Verfassung. Eine solche Unabdingbarkeit, die auf der Grundlage der Trennung des staatlichen Bereichs und der Individualsphäre beruht, würde sich schlecht mit der Vorstellung der hierarchisch-autoritären Ganzheit vertragen. Nach den meisten Verfassungen der spanischamerikanischen Länder hat der Kongreß als theoretisch oberstes Organ die Befugnis, alle Verhältnisse durch Gesetz zu ordnen, Gesetze zu interpretieren und aus festgelegten Ausnahmegründen auch namentlich aufgezählte Grundrechte wie Presse-, Versammlungsfreiheit, Briefgeheimnis oder Freizügigkeit zeitweise zu suspendieren. In der Praxis sind die Grundrechte durch das Überwiegen der meist diktatorischen Charakter tragenden Exekutive noch weit weniger garantiert ${ }^{71}$. Die neueste Entwicklung beginnt aber auch, dem Schutz der Grundrechte größere Aufmerksamkeit zu widmen. Interessant in dieser Hinsicht ist, daß das sogenannte "Amparo"-Verfahren in einer Reihe von Ländern eingeführt worden ist, nachdem es zunächst in México ausgebildet worden war. Es beruht auf der Erkenntnis, daß eine verfassungsmäßige Garantierung von Grundrechten erst dann ihren Sinn erhält, wenn die Individualrechte auch gerichtlich erzwingbar sind ${ }^{2}$.

Problematik und Unterschiedlichkeit gegenüber den Vorstellungen und Modellen des westlichen Liberalismus beruhen nicht zuletzt auf einem eigenen Begriff von Freiheit, wie er in Spanischamerika entstanden ist. Die Grundlagen hierfür haben sich in der Kolonialepoche gebildet. Formell staatsrechtlich waren die Bewohner Amerikas Untertanen der Krone, praktisch waren sie jedoch weitgehend sich selbst überlassen; das Kolonialregime hat kein gemeinschaftsverpflichtendes Verhalten $\mathrm{zu}$ erzeugen vermocht. Königliche Anordnungen wurden vielfach nur gebührend zur Kenntnis genommen, aber nicht befolgt. Mit der Formel „Se acata, pero no se cumple“ wurden üblicherweise königliche Erlasse beiseite glegt, wenn sie nicht genehm oder den Erfordernissen nicht passend erschienen ${ }^{73}$.

\footnotetext{
70 Gerhard Masur, Nationalism in Latin America. - New York/London 1966. - S. 239.

71 Glen Dealy, Prolegomena on the Spanish American Political Tradition, in: The Hispanic American Historical Review, Vol. XLVIII, Nr. 1, Febr. 1968. - S. $53 \mathrm{f}$.

72 Siehe Hans Christoph von Rohr, der argentinische Amparo-Prozeß. - Diss., Bonn 1969, besprochen in VRU 1969, S. 501; Hans-Rudolf Horn, Das Amparo-Verfahren in Mexiko. In: VRU 1969, S. $286 \mathrm{ff} .-$ J. Miranda, vgl. Anm. 64), S. $286 \mathrm{ff}$. - Reyven, Grundrechtsschutz in Argentinien, VRU 1970, S. $179 \mathrm{ff}$. 73 O. C. Stoetzer, vgl. Anm. 43). - S. 86.
} 
Eine solche weitherzige Behandlungsweise entspricht dem spanischen Individualismus, der in der Weite des Raumes eine spezifisch egoistische Ausprägung erfuhr. Hinzu kommt das dem Spanier eigene Gefühl für ein natürliches Widerstandsrecht gegenüber Despotismus und ungerecht erscheinenden Anordnungen, das in den Kolonien schon zu Revolten geführt hatte, so z. B. in Ecuador wegen ungerechter Besteuerungen. In Spanischamerika hatte sich eine Freiheit vo m Staate entwickelt, mit rücksichtsloser Ausnutzung individueller Chancen, aber wiederum nicht im Sinne der liberalen Theorie, die davon ausgeht, daß sich der einzelne seine irdische Freiheit durch Arbeit und unternehmerische Tüchtigkeit erwirbt. Dem spanischen Element fehlt diese unmittelbare Verknüpfung von Freiheit und nützlicher werteschaffender Arbeit.

Auf mangelndes Verständnis des Spaniers für gemeinschaftskonformes soziales Verhalten hat Ramón Menéndez Pidal hingewiesen ${ }^{74}$. Fernando Guillén Martínez hat es mit "falta de solidaridad social“ gekennzeichnet, als einen „individualismo feroz"75. Für den Spanier - und sicherlich noch mehr für den Spanischamerikaner - gilt als „ideal jurídico“: „que todos los Españoles llevasen en el bolsillo una carta foral con un solo artículo: Este Español está autorizado para hacer lo que le da la gana“. Die von der Krone vergebenen Sonderrechte (fueros) bedeuteten die „negación de la ley“. „La Nación española profesa poco afecto a los códigos"76. In Spanischamerika waren die Tendenzen einer zügellos materialistischen undisziplinierten Freiheit sicherlich auch dadurch verstärkt worden, daß sie einen psychologischen Ausgleich bildeten zu der von der Krone versagten politischen Gleichstellung der Kreolen mit den Spaniern.

Dieser Freiheit für eine verhältnismäßig kleine Zahl von Bevorzugten standen die Unfreiheit, in der die unteren Schichten gehalten wurden, und die radikale Ungleichheit in der Behandlung gegenüber - eine verhängnisvolle Widersprüchlichkeit des spanischamerikanischen Systems, die sich in den selbständigen Staaten nicht nur erhalten, sondern zunächst noch krasser ausgeprägt hat.

Der Prozeß der Angleichung an die Notwendigkeiten der modernen industriellen Gesellschaft wird auch hier zwangsläufig einen Sinneswandel, eine Änderung der traditionellen Mentalität herbeiführen, sei es durch innere Anpassung, durch Umerziehung oder durch heilsamen staatlichen Zwang, wie er im Bereich der Steuermoral bereits fühlbar wird. Auch für die spanischamerikanische Bevölkerung gilt, daß ein moderner Staat eine Fülle von öffentlichen und infrastrukturellen Aufgaben zu bewältigen hat, für die die Mittel primär nur von den Beteiligten selbst aufgebracht werden können.

\section{Exekutive und Gewaltentrennung}

Ein weiteres ebenso entscheidendes wie problematisches Merkmal des heutigen Spanischamerika ist das Überwiegen der Herrschaftsgewalt in Form offener oder verdeckter Diktaturen. Ein praktischer Grund liegt bereits in der Auswirkung einer so wenig gebundenen und staatskonformen Freiheit: einer so schwach aus sich heraus funktionierenden Lebensordnung muß zwangsläufig eine betont starke Herr-

\footnotetext{
74 Ramón Menéndez Pidal, Die Spanier in der Geschichte. - Deutsch beim Verlag Hermann Rinn, München 1955 . - S. 42, 47, 54, 87.

75 F. Guillén Martínez, vgl. Anm. 52). S. 39.

76 Angel Ganivet, Donde está España? - Auszugsweise in: España como Preocupación, Hrsg. Dolores Franco. Ediciones Guadarrama, Madrid 1960, 2. Aufl. - S. 308.
} 
schaftsgewalt gegenüberstehen, da sonst ein Abgleiten des öffentlichen Lebens in Anarchie droht. Die Ordnung kann offenbar im allgemeinen nur durch Herrschaftsausübung diktatorischen Charakters aufrechterhalten werden, wobei die verfassungsmäßigen Spielregeln immer wieder außer Kraft gesetzt werden.

Aber auch grundsätzlich sind die Auffassungen verschieden. Die klassische liberale Auffassung geht davon aus, daß sich die private Sphäre und der Staatsbereich getrennt gegenüberstehen. Der Staat wird mißtrauisch als "gesellschaftlicher Zwangs- und Unterdrückungsapparat "77 angesehen, er darf nur mit geringst möglichen Befugnissen ausgestattet werden. Ein solches Verhältnis zwischen Bürger und Staat hat Wilhelm von Humboldt in seinen "Ideen zu einem Versuche, die Grenzen der Wirksamkeit des Staates zu bestimmen" dargestellt. Der Staat ist dabei bestenfalls ein notwendiges Übel, ein "Nachtwächterstaat". Auch schon in der liberalen Praxis hat sich eine Konstruktion auf solcher Grundlage als zu unreal erwiesen; man hatte in der ersten Begeisterung den Menschen für freiheitsliebender gehalten als er tatsächlich ist ${ }^{78}$.

Das spanischamerikanische Denken beruht auf einer Vorstellung der Einheit von Staat und Bevölkerung, in der die Regierung verpflichtet ist, auch für die Beachtung der Rechte des einzelnen zu sorgen. Die primäre Verpflichtung liegt bei der Regierung, wie diese auch primär verantwortlich ist für die Regelung der Ordnung im öffentlichen Bereich, ohne daß es prinzipiell so etwas gibt, wie den Vorgang der autonomen Ordnung aus der "prästabilierten Harmonie“ der klassischen liberalen Theorie. Außerdem liegt im hispanischen Denken ein ausgeprägter Sinn für die Konzentrierung der Macht in einer Person. Diesem Denken lag daher die Form der Präsidialdemokratie am nächsten, das von allen Verfassungen dieser Länder übernommen wurde. Es ist kennzeichnend, daß sich das von Uruguay praktizierte Experiment einer kollegialen Regierungsspitze, nach der Verfassung von 1952, rasch als unbrauchbar erwies. In der Person des vom Volke direkt gewählten Präsidenten wird die Exekutivgewalt zusammengefaßt; er ist zugleich Repräsentant, Ministerpräsident und oberster Kriegsherr. Bezeichnend sind die personalistischen Formulierungen, mit denen dem Präsidenten die Ausübung der Regierungsgewalt übertragen wird. In der Verfassung von México von 1917, Art. 80 (gleichlautend in der Verfassung von 1857, Art. 75), heißt es: „Se deposita el ejercicio del Supremo Poder Ejecutivo de la Unión en un solo individuo, que se denominará „Presidente ...“. Die Verfassung von Perú sagt in Art. 134: „El Presidente . . . personifica la Nación“. Die Verfassung von Chile, Art. 60, nennt den Präsidenten den obersten Chef der Nation.

Die Verfassungen enthalten außerdem Regelungen über die Handhabung des Ausnahmezustandes, die an sich schon "con suma generosidad"79 formuliert worden $\operatorname{sind}^{80}$ und die zudem üblicherweise sehr weitherzig angewandt werden. Hieraus ergibt sich eine nur wenig eingeschränkte Machtbefugnis des Präsidenten; für México hat Gonzalez Casanova festgestellt: „El poder presidencial no tiene lími-

\footnotetext{
77 Ludwig von Mises, Artikel Liberalismus II, in: Handwörterbuch der Sozialwissenschaften, Bd. 6, S. 561, - Göttingen 1959.

78 Carl Joachim Friedrich, Artikel Demokratie, in: Handwörterbuch der Sozialwissenschaften, Bd. 2, S. 561. - Göttingen 1959.

79 C. Pisano Fischer, vgl. Anm. 1). - S. 138.

80 S. Art. 175 (Verfassung von El Salvador), Art. 44, Ziff. 13 - Chile, Art. 107 - Honduras, Art. 52, 53 - Panamá, Art. 79 - Paraguay, Art. 70 - Perú, Art. 121, Ziff. 7 - Costa Rica.
} 
tes. ${ }^{81 " ~ E s ~ k a n n ~ n i c h t ~ v e r w u n d e r n, ~ w e n n ~ e i n e ~ s o l c h e ~ p e r s o n a l i s t i s c h e ~ H e r r s c h a f t s-~}$ ausübung vom liberalen Standort aus als undemokratisch gekennzeichnet worden ist $^{82}$.

Dieses hierarchisch-personalistische Prinzip entspricht auch dem Empfinden der Bevölkerung. Solange der Machthaber durch Leistungen imponiert, kann er auf den Konsens der Bevölkerung rechnen, der ein Verhältnis zur Person des Regierenden mehr liegt als zu einer abstrakten Institution. Diese Einstellung ist gefühlsbetont und neigt zu utopischen Wunschvorstellungen von dem tüchtigen Führer; hierher gehört, daß in den Verfassungen häufig die Qualitäten genannt sind, die von dem höchsten Würdenträger erwartet werden ${ }^{83}$.

Das personalistische Prinzip beherrscht auch heute noch weitgehend das Parteiwesen. Auch hier folgen die Anhänger dem politischen Caudillo, dem Patrón, dem starken Mann, und nicht einem abstrakten Parteiprogramm ${ }^{84}$. „Das typische Element der angelsächsischen Demokratie, das Parteisystem, verändert sich gänzlich bei der Verpflanzung nach Spanischamerika, wo es Gestalt nahm in Familien- und Geschlechter-Rivalitäten "85. Ein neues Beispiel eigener Art bietet in México die herrschende „Partido Revolucionario Institucional“ (PRI) ${ }^{86}$. Nur aus ihr gehen seit einigen Jahrzehnten die mexikanischen Präsidenten hervor, sie gibt dem politischen Leben in México die sonst so sehr vermißte Stabilität; sie hat eine gewisse Einigung aller Mexikaner und ein gewisses Maß an Mitwirkung erreicht und die politische Zerrissenheit überwunden. Gonzalez Casanova sieht ihre Hauptfunktion darin, den Caudillo, den Caziquen und Patrón zu entmachten und deren Tatkraft sinnvoll in das Staatsleben einzuordnen ${ }^{87}$. Eine solche gemäßigte und irgendwie gebändigte diktatorische Gewalt des Präsidenten ist so etwas wie eine „forma necesaria“, ein "régimen político normal de América Latina“88, womit der bisherige schon fast als normal zu bezeichnende Zustand des ständigen Wechsels durch immer neue "pronunciamientos“ überwunden wird.

Das liberale Prinzip der Gewaltentrennung, das aus dem Mißtrauen gegen die Staatsgewalt zu einem Dogma wurde, muß gegenüber den ganz auf die Person des charismatischen Führers abgestellten Auffassungen fremd bleiben. Eine rationale und mechanistische Aufspaltung der Herrschergewalt widerspricht im tiefsten der spanischamerikanischen und katholischen Auffassung von der organischen Ganzheit und Einheit der staatlichen Funktionen. Die Gewalt muß zwar begrenzt werden, aber niemals geteilt ${ }^{89}$. Die Begrenzungen der Macht erfolgen im personalistischen Sinne, und zwar durch Festlegung persönlicher Qualitäten oder durch Erschwerung einer Wiederwahl.

81 P. Gonzales Casanova, vgl. Anm. 1). - S. 27.

82 Glen Dealy, vgl. Anm. 71). - S. 52.

83 id., S. 55.

84 Jacques Lambert, Latin America, Sozial Structure and Political Institutions. Berkeley and Los Angéles 1967. - S. $359 \mathrm{f}$

85 Manuel Fraga Iribarne, Sociedad Política y Gobierno en Hispanoamérica. Madrid 1962. S. 92.

86 Ausführliche Darstellung in: Robert K. Furtak, Die Partido Revolucionario Institucional und die politische Stabilität Méxicos. - Hamburg 1969.

87 P. Gonzalez Casanova, vgl. Anm. 1). - S. 29 f

88 C. Pisano Fischer, vgl. Anm. 1). - S 127.

89 Vgl. hierzu: Glen Dealy, Anm. 71), S. 46. Felipe Tena Ramirez, Derecho Constitucional Mexicano. 8. Aufl., México 1967. - S. 210 f.' 


\section{Schlußbetrachtungen}

Ziel dieser Untersuchung ist es, einige Gedanken zu skizzieren zur Problematik und zu den Möglichkeiten einer stärkeren Demokratisierung in den spanischamerikanischen Ländern. Zusammenfassend läßt sich feststellen:

a) Für eine zeitgemäße und der modernen industriellen Gesellschaft konforme Entwicklung ist eine echte nicht nur als Tarnung vorhandene Demokratisierung nötig, sie ist sogar die unerläßliche Voraussetzung. Hierzu gilt es, die in der spanischamerikanischen Tradition liegenden Hemmnisse ebenso zu erkennen wie diejenigen Gesetzlichkeiten, die Spanischamerika grundsätzlich von der Mentalität der Liberal-Demokratie unterscheiden.

b) Demokratisierung meint die Eröffnung der Beteiligung aller Bevölkerungskreise am öffentlichen Geschehen, die Integrierung aller Bevölkerungsteile auf der Grundlage von Gleichheit und sozialer Gerechtigkeit. Hierzu ist die aktive Anteilnahme aller Kreise zu erwecken, die traditionelle Apathie und Resignation zu überwinden. Voraussetzung ist die Auflösung der starren Gesellschaftsstruktur mit der traditionellen Verquickung der oligarchisch-diktatorischen Machtverhältnisse.

c) Die hierarchisch-autoritären Elemente sind als Erbschaften der vorcolumbischen und der kolonialen Epochen und der hispanischen Mentalität so tief verwurzelt, daß eine Demokratisierung „von unten“ vorerst nicht zu verwirklichen sein wird. Der Volkswille aus eigener Antriebskraft hat keine Tradition. Allerdings zeigen sich bemerkenswerte Ansätze im Zuge der Bildung eines Mittelstandes und eines nationalen Selbstbewußtseins. Eine Entwicklung, wie sie der westeuropäische Liberalismus im Kampfe gegen den Absolutismus gebracht hat, dürfte sich in Spanischamerika aber nicht wiederholen können.

d) Spanischamerika wird eigene Herrschafts- und Lebensformen entwickeln müssen, und zwar nicht auf der Grundlage des egalitären Denkens, sondern aus eigenen Vorstellungen gemäßigt-hierarchischer Art. Staat und Individualbereich stehen sich nicht a priori feindlich gegenüber, sondern bilden eine ideelle Ganzheit. Demokratie wird daher nicht nur "von unten“, sondern wesentlich "von oben" durchgeführt werden müssen. In diese Richtung weisen alle bisherigen Beispiele, wie México, Cuba oder Perú. „Aufgeklärten“ Militärregierungen fallen entscheidende Aufgaben zu; sie haben allerdings den Nachteil, daß sie keine kontinuierlich stabilisierende Staatsform darstellen. Sobald die "auctoritas“ schwindet, bleibt nur noch die "potestas" - und dann droht das nächste "pronunciamiento". Der bisherige normale Staat leidet an „insuficiencia“, seine Struktur ist „imperfecta o deficitaria“ - er war bisher eher ein „obstáculo o un débil aliado del proceso de desarrollo"90.

e) Eine die Kontinuität garantierende Herrschafts- und Lebensform kann nur eine Mischung von demokratischen Zügen im Sinne einer allgemeinen Liberalität mit autoritärer Führung sein. Die spanischamerikanische Spielart des Liberalismus hat andere Ursprünge und geistige Voraussetzungen als die westeuropäisch-nordamerikanische. Dies gilt für den politischen wie für den wirtschaftlichen Liberalismus.

90 Marcos Kaplan, vgl. Anm. 7). - S. 110, 113. 
Das Verfassungsmodell der Vereinigten Staaten von Amerika taugt prinzipiell dafür nicht. Es werden sich eigene Formen entwickeln müssen mit einer Mischung von personalistischen und institutionellen Elementen. Demokratie muß eine „überpersonale Kontinuität" ${ }^{\text {"91 }}$ herstellen, um allzu personalistische Machtausübung zu bändigen. Verfassungen sind nicht mehr nur Deckmantel diktatorischer und reaktionärer Herrschaft - sie müssen als institutionelle Leitlinie integrierende Funktionen ausüben. Eine eigene spanischamerikanische Entwicklung könnte zu Formen führen, die mit "k onstitutionelle r Dikt a t u r "92, wenigstens für die bevorstehenden Zeiten des Abbaus unzeitgemäßer Traditionen, gekennzeichnet werden könnten. Ein Beispiel einer solchen Staatsform bietet México mit einer seiner Mentalität gemäßen Verfassung als anerkannter Institution und zugleich mit der kontinuierlichen Herausstellung einer autoritären Führungsspitze ${ }^{93}$, ohne an dieser Stelle auf die Problematik auch dieser Lösung einzugehen. f) Entscheidend ist auch das Verhalten der Welt gegenüber Spanisch- bzw. ganz Lateinamerika, d. h. die Einsicht in die eigene Art dieses amerikanischen Teilkontinentes und die Anerkennung des Bestrebens, als "personalidad propia die América Latina“ gewürdigt zu werden auf der Grundlage eines "profundo respecto a la identidad hispanoamericana humana y cultural "94 und nicht mehr als eine Art Anhängsel an die USA. Vor allem auch die USA selbst müssen ihr Verhältnis zu Spanischamerika überprüfen gegenüber einem Nachbarn, der anders als bisher seine eigene Position in der Welt einnehmen will. Neben der Belastung durch die seitherige Interventionspolitik ist es im Grunde der ideologische Gegensatz, der das Verhältnis der beiden Teile Amerikas so krisenhaft sein läßt ${ }^{95}$.

g) $\mathrm{Ob}$ die Entwicklung durch offene Revolutionen beschleunigt werden kann oder einem längerfristigen heute schon nicht mehr aufzuhaltenden Prozeß unterliegen wird, kann nur die Geschichte lehren. Auch die cubanische Revolution kam nicht „von unten“, sondern wurde unter günstigen Umständen durch eine Gruppe von Intellektuellen verwirklicht mit dem Ergebnis einer autoritär-hierarchischen Diktatur eines charismatischen Caudillos hispanischer Prägung. Nach Lage der Dinge wird eine Mitwirkung des Militärs unerläßlich sein. Der Weg über das Guerillatum führt offenbar eher zu Störungen als zu echten Lösungen — allerdings können die Motive idealistischer Guerilleros als Herausforderung nicht ernst genug genommen werden.

h) Für das Erwachen eigenen Selbstbewußtseins sind wissenschaftliche Erkenntnisse erforderlich, wofür insbesondere seit dem Zweiten Weltkriege bereits erhebliche Voraussetzungen geschaffen wurden. Hier muß vor allem die Tätigkeit der UN-Kommission für Latein-Amerika (Comisión Económica para América Latina - CEPAL) unter der Leitung von Raúl Prebisch im Ringen um eigene wirtschaftliche und wirtschaftspolitische Konzeptionen erwähnt werden. Bezeichnend ist, daß neben soziologischen Forschungen auch eine philosophische und politologische Er-

91 Konrad Hesse, Grundzüge des Verfassungsrechts der Bundesrepublik Deutschland. - 2. Aufl. Karlsruhe 1968. - S. 55.

92 Eine solche "contradictio in adjecto" müßte ebenso anwendbar sein wie diejenige des eingebürgerten Begriffs der "konstitutionellen Monarchie".

$93 \mathrm{Vgl}$. Hans-Rudolf Horn, Revolution und Verfassung. Ubersee-Verlag Hamburg 1919 („Schriftenreihe des Instituts für Iberoamerika-Kunde“, Heft 10).

94Dr. Pablo Latapí, auf der Tagung des Ibero-Amerika Vereins und des Instituts für IberoamerikaKunde in Hamburg, Oktober 1969.

95 Zum Problem der Beziehungen der USA zu Lateinamerika, auf die in diesem Rahmen nicht näher eingegangen werden kann, siehe z. B.:

Peter Schenkel, Die ideologische Herausforderung Lateinamerikas durch die cubanische Revolution, in: Idee und Wirklichkeit in Iberoamerika, vgl. Anm. 8, insbesondere S. $100 \mathrm{ff}$., S. $117 \mathrm{f}$. 
forschung des eigenen „ser histórico“ in den Vordergrund tritt ${ }^{96}$. Der Prozeß der „emancipación mental“97, der 1810-1825 versäumt wurde, wird jetzt nachgeholt und damit wird ein neues Verhältnis zu den historischen Erbschaften wie gleichermaßen auch zur industriellen und technologischen Epoche von heute gesucht. Diese Entwicklung ist nicht denkbar ohne die Bildung eigener Staatsformen.

96 Hierzu: Carlos Fortín, Las Posibilidades del estudio político comparado en América Latina. In: Perspectivas ..., vgl. Anm. 7), S. $93 \mathrm{ff}$.

Und: Marcos Kaplan, La Ciencia Política Latinoamericana en la Encrucijada, In „Aportes ${ }^{\alpha}, \mathrm{Nr} .{ }_{16}$ April 1970, S. $130 \mathrm{ff}$.

97 Leopoldo Zea, vgl. Anm. 57), S. 11. 